\title{
Dysfunctional Lens Syndrome
}

Running title: Dysfunctional lens syndrome

\section{Mohammad Reza Sedaghat, MD; Hamed Momeni-Moghaddam, MSc²; Shehzad A. Naroo, PhD ${ }^{3}$; Hossein Ghavam-Saeedi, MD ${ }^{1}$; Alireza Vahedi, MD $^{1}$}

1. Eye Research Center, Mashhad University of Medical Sciences, Mashhad, Iran.

2. Department of Optometry, School of Paramedical Sciences, Mashhad University of Medical Sciences, Mashhad, Iran.

3. Aston University, Ophthalmic Research Group, School of Life and Health Sciences, Aston Triangle, Birmingham B4 7ET, United Kingdom.

Corresponding author: Hamed Momeni-Moghaddam, MSc.

Postal address: Mashhad Optometry Department, Parastar 2 Str., Ahmadabad Blvd., Mashhad, Iran.

E-Mail: hmomeni_opt@yahoo.com

Tel: +989155337952 


\begin{abstract}
This report is related to the pre- and post-operative findings of a case with dysfunctional lens syndrome. A 45-yearold male patient presented with the chief complaint of poor visual quality; uncorrected visual acuity 20/40 in the right eye, best spectacle corrected visual acuity $20 / 25$ in the right eye with refraction Plano/-1.50×80 (SE= $-0.75 D)$. Preand post-operative root-mean-square (RMS) of total higher-order aberration in the entire eye, the internal optics and the cornea were $0.350 \& 0.257,0.311 \& 0.236$ and $0.214 \& 0.191$ micron $(\mu)$, respectively. Also, the magnitude of preoperative total, internal and corneal coma was $0.254 \mu \times 222^{\circ}, 0.274 \mu \times 242^{\circ}$ and $0.097 \mu \times 131^{\circ}$ and postoperative values were $0.170 \mu \times 162^{\circ}, 0.131 \mu \times 177^{\circ}, 0.054 \mu \times 125^{\circ}$, respectively. These results show that sometime the HOAs of the internal optics, mainly crystalline lens, are not compensated by the cornea and this may cause visual discomfort in the absence of any significant cataract, this situation is known as dysfunctional lens syndrome (DLS).
\end{abstract}

Key Words: Crystalline lens; Dysfunctional lens syndrome; Higher-order aberration; Internal aberration 


\section{Introduction}

Visual function is influenced by environmental, optical and neural factors. Retinal image and visual quality are affected by light scattering at the optical media, diffraction at the pupil border and optical aberrations (low and higherorders) of the eye [1].

Higher-orders aberrations (HOAs) can be responsible for glare, haloes, night vision problems, decreased contrast sensitivity, discomfort in daily activities and consequently reduced quality of life [2]. Lenticular high-orders aberrations are one the causes of increased ocular HOAs after the age of 50 years [3]. Assessment of HOAs in senile cataract showed that opacity location has different effects on the ocular aberrations so that nuclear and cortical cataracts are mostly associated with higher amounts of spherical and coma aberrations, respectively [4].

Sometimes patient complains of reduced visual quality or quality of life related to vision despite normal vision or VA so that the patient is not satisfied to carry-out their usual activities. One reason may be the increased HOAs of the internal ocular optics, especially crystalline lens, known as dysfunctional lens syndrome (DLS). Here is the pre- and post-operative findings of a case with dysfunctional lens syndrome.

\section{Case Presentation}

A 45-year-old man complained of poor quality of vision, driving and night vision problems. His uncorrected visual acuity (UCVA) and best corrected visual acuity (BCVA) were 20/40 and 20/25, respectively with a refraction Plano/$1.50 \times 80^{\circ}(\mathrm{SE}=-0.75 \mathrm{D})$ in the right eye. History of ocular surgery, ocular trauma, ocular and systemic diseases was negative.

The corneal topography using TMS-5 (TOMEY Corporation, USA) was normal pattern and indices. Slit-lamp examination showed transparent cornea and crystalline lens without any sign of anterior or posterior lenticonus and tilt or decentration. Tear film assessment using slit-lamp did not show any abnormality. Also, dilated fundus examination was normal. IOP was $14 \mathrm{mmHg}$ using non-contact tonometer (Topcon, Japan).

The Baseline wavefront analysis using i-Trace aberrometer (Tracey Technologies, Houston, TX) showed a significant amount of total HOAs $(\mathrm{RMS}=0.350 \mu \mathrm{m})$ that is mostly due to the internal optics $(\mathrm{RMS}$ total HOAs $=0.311 \mu \mathrm{m})$ than the cornea $(\mathrm{RMS}$ total HOAs $=0.214 \mu \mathrm{m})$. Assessment of the magnitude of any given aberration represented that the total coma $\left(0.254 \mu \times 222^{\circ}\right)$ was the possible cause of patient's symptoms and was the result of internal optic mostly crystalline lens $\left(0.274 \mu \mathrm{m} \times 242^{\circ}\right)$ that had not compensated or balanced by the cornea $\left(0.097 \mu \mathrm{m} \times 131^{\circ}\right)$. (Figure 1$)$ 
""Figure 1""

There are different management options for this condition that one used for the current patient was dysfunctional lens exchange in which the crystalline lens is removed and replaced with an intraocular lens implant. The post-operative assessments were UCVA of 20/20, refraction $+0.25 /-1.00 \times 85(\mathrm{SE}=-0.25 \mathrm{D})$ in the right eye, and considerable clinical improvement in the entire eye total HOAs $(0.257 \mu \mathrm{m}$ vs. $0.350 \mu \mathrm{m})$ and the internal optics total HOAs $(0.236 \mu \mathrm{m}$ vs. $0.311 \mu \mathrm{m})$ compared to the pre-operative values. In addition, there was significant reduction in the entire eye and internal optics coefficients of coma. (Figure 2)

"'Figure 2""

\section{Discussion}

These findings emphasize that the crystalline lens is possible source of patient's discomfort in the absence of cataract, hence, diagnosis is the dysfunctional lens syndrome (DLS) which refers to diminished visual performance secondary to the effects of HOAs or occasionally senile subtle loss of transparency of crystalline lens. Although HOAs are accounted for about $10 \%$ of the total ocular aberrations, but they have a significant effect on the retinal image quality. This description highlights the diagnostic difference between functional and refractive origins of visual complaints $[5]$.

In DLS, the effect of lenticular optical aberrations (primarily spherical or coma aberrations) not neutralized or balanced by the corneal aberrations of the cornea, which increasingly reduce the quality of vision and may produce diurnal and nocturnal visual problems [6].

Other causes of poor visual quality such as light scattering in any component of the optical system, light diffraction, and changes in the normal structure of retina should be considered in the patient's evaluation. It is important to emphasize that although the patients' symptoms are such as those described by patients with cataract, but the results of examination did not show a cataract. To confirm diagnosis and provide substantial objective evidence of functional 
visual impairment, taking a careful detailed history of patient's complaints along with contrast sensitivity testing and wavefront aberrometry of the eye seem helpful.

Other instruments in addition to the iTrace (Tracey Technologies, Houston, TX) which can be used in the differential diagnosis or contributing to the diagnostic process of DLS via more analysis of the crystalline lens are such as HD Analyzer (Visiometrics, Barcelona) a double-pass wavefront device that measures light scatter which provides an optical scatter index (OSI) and a simulated visual acuity reading; Nidek/Marco OPD-Scan III (NIDEK CO., LTD.) with the ability to separate the internal and external aberrations; Oculus Pentacam (Oculus Optikgeräte GmbH, Wetzlar, Germany) to check the densitometry on different parts of crystalline lens on the Scheimpflug image [7]. Based on the provided three-stage classification system for DLS, the present case was in the stage I. Stage I often occurs in the 40 s with a clear and colorless but stiffer crystalline lens along with the gradual loss of focusing ability at near distances, Stage II in the 50s and 60s along with turning yellow and slight haziness of crystalline lens, minor problems with night vision and requiring more light for reading and Stage III is characterized by cataract, secondary visual impairment and considerable limitation of patient's daily activities [8].

In conclusion, DLS is characterized by several clinical findings gradual decrease of crystalline lens transparency localized in cortical or nuclear areas or both, gradual loss of accommodative ability and increased optical aberration profiles in particular spherical and/or coma aberrations. In this situation, if the symptoms limit the patient' performance, functional not refractive lens surgery is indicated. In other word, a patient with impairment of visual function and daily living activities despite having a relatively good visual acuity is a suitable candidate for functional lens surgery.

Source(s) of support: The authors indicate no funding support.

Acknowledgement: The authors would like to thank the subject and personals of Sedaghat eye clinic.

Conflicts of Interest of each author/ contributor: The authors have no conflicts of interest to declare.

Patient Consent: The patient has consented to the submission of the case report for submission to the journal. 


\section{References}

1. Alió JL, Schimchak P, Negri HP, Montés-Micó R (2005) Crystalline lens optical dysfunction through aging. Ophthalmology 112(11):2022-29.

2. Lyall DA, Srinivasan S, Gray LS (2013) Changes in ocular monochromatic higher-order aberrations in the aging eye. Optom Vis Sci 90(9):996-1003.

3. Fujikado T, Kuroda T, Ninomiya S, Maeda N, Tano Y, Oshika T, Hirohara Y, Mihashi T (2004) Age-related changes in ocular and corneal aberrations. Am J Ophthalmol 138(1):143-6.

4. Rocha KM, Nosé W, Bottós K, Bottós J, Morimoto L, Soriano E (2007) Higher-order aberrations of age-related cataract. J Cataract Refract Surg 33(8):1442-6.

5. Lombardo M, Lombardo G (2010) Wave aberration of human eyes and new descriptors of image optical quality and visual performance. J Cataract Refract Surg 36(2):313-31.

6. Roorda A, Glasser A (2003) Wave aberrations of the isolated crystalline lens. J Vis 4(4):250-61.

7. Mello GR, Rocha KM, Santhiago MR, Smadja D, Krueger RR (2012) Applications of wavefront technology. J Cataract Refract Surg 38(9):1671-83.

8. https://www.aao.org/eyenet/academy-live/detail/dysfunctional-lens-syndrome-educate-patients 
Figure legends:

Figure 1: Pre-operative wavefront analysis.

TRACEY

WF and CT Summary Display

\section{HOYA涫race Surgical Workstation}

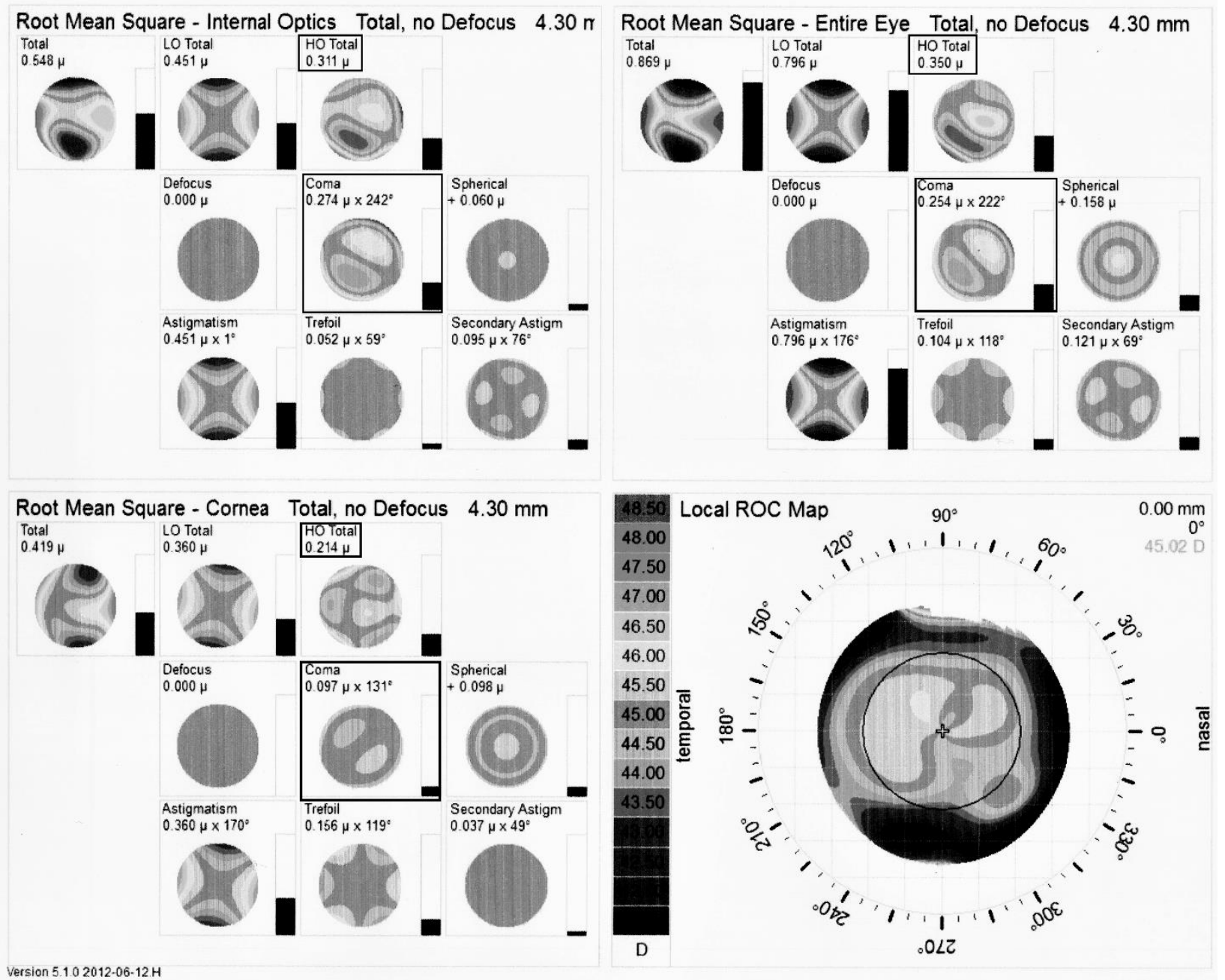


Figure 2: Post-operative wavefront analysis.

TRACEYO
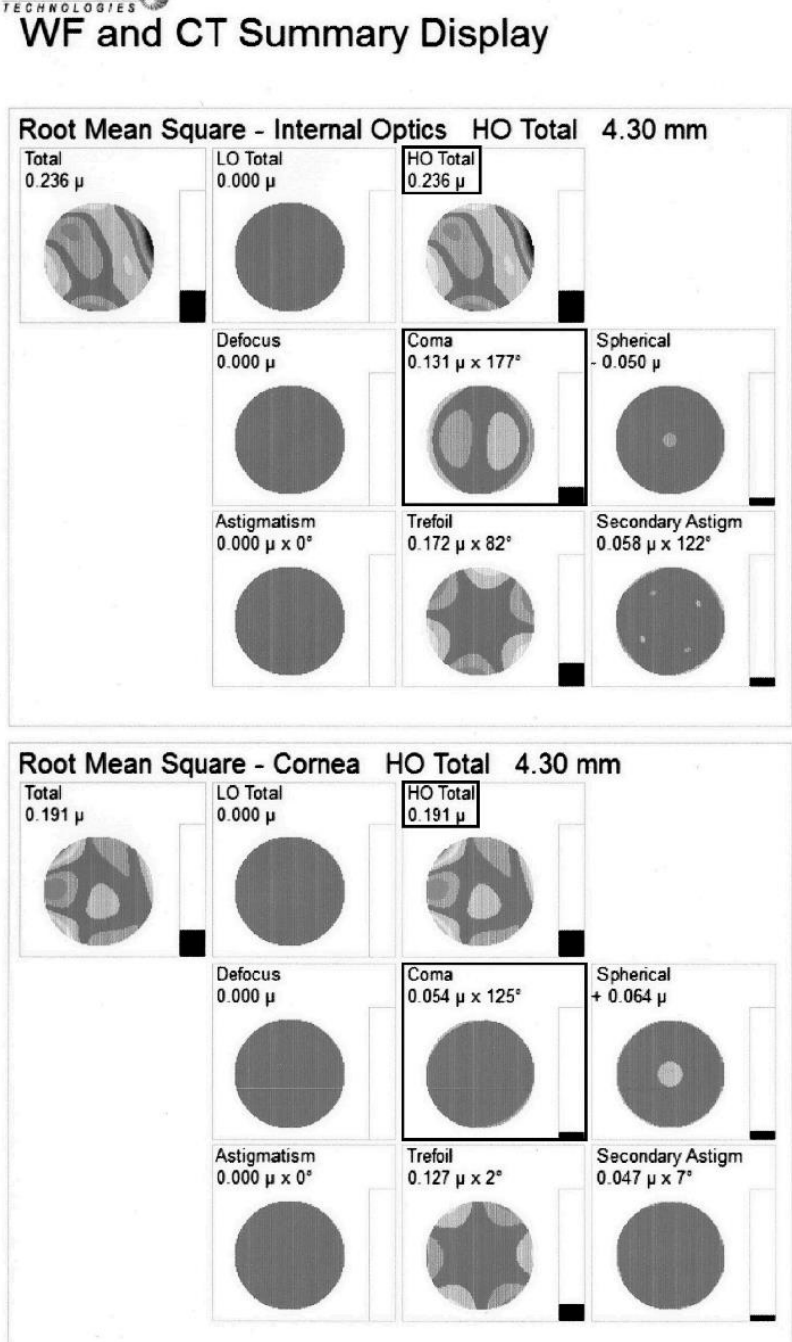

Version 5.1.0 2012-06-12.H

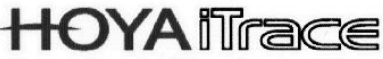 Surgical Workstation}

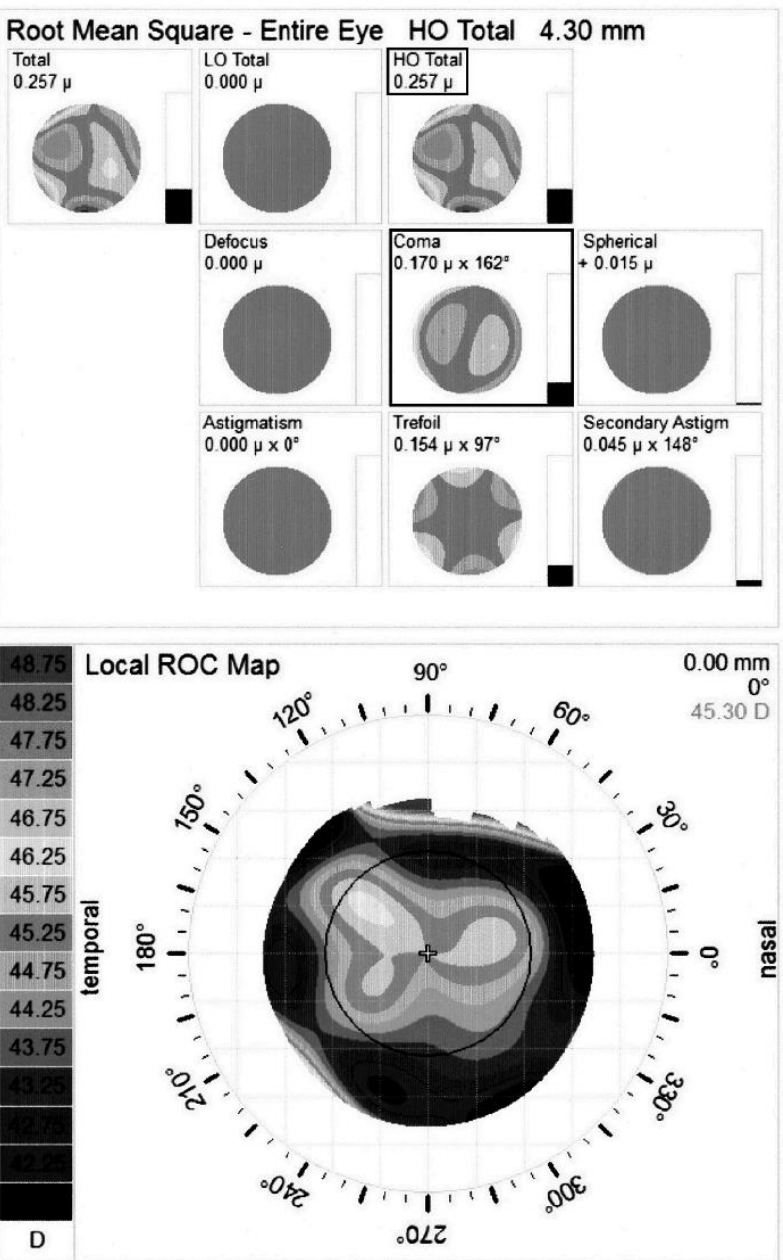

